\title{
Perspective
}

PERSPECTIVE Actualité en histoire de l'art

$2 \mid 2020$

Danser

\section{Faire danser la pensée}

\section{Georges Didi-Huberman}

\section{(2) OpenEdition}

\section{Journals}

Édition électronique

URL : https://journals.openedition.org/perspective/19845

DOI : 10.4000/perspective. 19845

ISSN : 2269-7721

Éditeur

Institut national d'histoire de l'art

Édition imprimée

Date de publication : 30 décembre 2020

Pagination : 10-16

ISBN : 978-2-917902-90-5

ISSN : $1777-7852$

Référence électronique

Georges Didi-Huberman, «Faire danser la pensée », Perspective [En ligne], 2 | 2020, mis en ligne le 30 juin 2021, consulté le 29 juillet 2022. URL : http://journals.openedition.org/perspective/19845 ; DOI : https://doi.org/10.4000/perspective.19845 
TRIBUWE

\title{
Faire danser la pensée
}

\author{
Georges Didi-Huberman
}

"Danser. Le voulez-vous ${ }^{1}$ ?... " (Tanzen. Wollt ihr das?...) Tels furent les tout derniers mots écrits par Nietzsche pour Le Gai Savoir. Les tout premiers avaient été pour dénoncer les philosophes ou les moralistes qu'il nommait, avec colère, les " doctrinaires de l'existence ${ }^{2}$ ". Danser, était-ce enfin avoir une chance d'exister sans la prison des doctrines ? Mais pourquoi convoquer ainsi la danse comme point d'orgue à ce grand livre de soulèvement ( "Nous sommes tous des volcans en croissance qui attendent l'heure de leur éruption ${ }^{3}$ ") ? Que devait-il se passer entre une dénonciation de toutes les ontologies figées dans leurs certitudes doctrinales et une telle invitation à danser ? Quel était l'enjeu ? Rien de moins, sans doute, que la mise en mouvement de notre pensée elle-même : sa mise en rythme et, donc, sa prise de risque pour quelque chose comme une nécessité d'émancipation.

" Danser. Le voulez-vous ?... » En soulignant le verbe, Nietzsche insistait clairement sur la notion de vouloir : cette fameuse "volonté de puissance " que, contrairement aux lectures triviales qui en eussent fait une intention de dominer, de posséder ou que sais-je encore, le philosophe définissait plutôt comme un pathos, une sensibilité à la force et de la force elle-même. Ce que Gilles Deleuze aura bien résumé par ces mots : "La volonté de puissance se manifeste comme un pouvoir d'être affectét. " Vouloir, ce n'était donc pas imposer ou, même, prendre quoi que ce fût. C'était donner : donner libre cours. Laisser aller l'existence, l'accompagner dans son libre déploiement. Vouloir danser, par conséquent, ne désignait rien d'autre que ceci : vouloir donner forme et temps, corps et mouvement, vouloir donner rythme à sa propre puissance d'être affecté.

Il est tout à fait vain de parler de la danse en général (prétendre à cette généralité semble, justement, une prétention typique des « doctrinaires de l'existence »). À chacun sa danse.

On danse comme on peut et, surtout, selon ce qu'on veut. La danse est comme les rêves : elle est tellement intime - et pourtant manifeste - que la danse des uns est souvent

1. Julio Palomar, José Tomás aux arènes de Las Ventas (détail), Madrid, le 18 mai 2001. regardée par les autres avec incompréhension, moquerie, dégoût quelquefois. On n'admire la danse de l'autre que si l'on est capable d'admirer le désir de l'autre. Voici par exemple 
un " désir tout autre " et tout à fait singulier (particulièrement pour quelqu'un qui, comme moi, ne "danse " que dans les bibliothèques en papillonnant de livre en livre, et passe une grande partie de sa vie assis à une table, un stylo à la main, essayant de faire danser les mots sur des feuilles de papier blanc).

Je veux parler d'un désir rare, en effet. Et profondément anachronique. Celui de vouloir danser la mort - et même de vouloir danser dans l'entre-deux infiniment risqué de deux morts, la sienne et celle d'un taureau sauvage. Un mot, donc, quelques mots ou bouts de phrases, sur la danse extrême du torero José Tomás. Ce que j'ai vu le 15 juin 2008 dans les arènes madrilènes de Las Ventas aura gravé en moi l'image même, intranquille, infiniment puissante et fragile, de ce que peut constituer, radicalement, une " puissance d'être affecté ". Tout a été dit, redit et contredit, en Espagne et ailleurs, sur José Tomás : petit bout d'homme que beaucoup appellent, éperdus, " maestro de maestros " (et à qui beaucoup de doctrinaires taurins auront longtemps cherché des poux dans la tête, avant de capituler devant sa grandeur). Nijinski, à l'évidence, fut bien moins respecté. J'ai vu, ce jour-là, José Tomás danser la mort en face - et surtout pas " marcher à la mort ", comme on le dit des persécutés, ni même "éviter la mort", comme on le dit des gens malins. Pour le dire un peu plus précisément : danser aux deux morts. Celle que le rituel taurin lui prescrivait de donner, et celle dont un monstre noir antédiluvien, irrésistiblement puissant dans sa volonté de tuer, le menaçait à chaque bribe d'instant.

Ce que j'ai vu ce 15 juin 2008, je l'ai bien sûr mal vu, n'étant pas de ces aficionados qui savent - par un art de la mémoire dont seuls les vieux cinéphiles, à ma connaissance, peuvent se prévaloir encore - faire de chaque instant d'une corrida quelque chose comme un impérissable monument de mémoire précise et ciselée, susceptible de gloses à l'infini. J'ai vu confusément. Du moins ai-je pressenti quelque chose comme un extraordinaire pas de trois. Au centre de tout : le temps de l'immobilité. Attendre sans broncher-aguantar: endurer, souffrir, patienter, résister -, et pourtant le fauve arrive de toute sa force. Ce n'est pas une immobilité d'être éternel ou plus solide que tout, mais au contraire une suspension fragile d'oiseau dans le vent contraire. Il tient pour l'instant. Ça passera ou ça cassera. Tension partout : le taureau charge, vingt-trois mille personnes font entendre qu'elles retiennent leur souffle, et pourtant cela tend à l'immobilité. Au mieux de tout, le temps de la caresse : moments de frôlements suaves, enchaînements de gestes devenant irréels parce que vus comme impossibles, tangentes de danger lorsque la corne passe si près et que la masse énorme peut tout renverser sur son passage.

Au pire : temps de la blessure. Mortelle, cela arrive, bien sûr. Et c'est cela que le danseur taurin accepte comme la condition d'existence de toute sa " puissance d'être affecté ". Plusieurs fois, ce jour-là, j'ai cru le voir transpercé de part en part. Mais non, il avait mis son corps entre les cornes mortelles, comme sur cette photographie où dans les mêmes arènes, en 2001, il avait été soulevé par le taureau, fétu de paille et de paillettes (fig. 1). Il fut bien, ce jour de 2008, gravement blessé par trois fois, comme le rapporta Jacques Durand dans l'une de ses irremplaçables chroniques : "Une statue de pierre couverte de sang. Le sien, celui du toro. Il a trois coups de cornes dans le corps, un de vingt centimètres en haut de la cuisse droite, un dans le genou, un dans le mollet, et... rien. Il torée comme si de rien n'était. [...] Pas de tour de piste. Il est blanc, son habit est rouge de sang, il a un petit sourire. Il part à pied à l'infirmerie. Anesthésie, opération ${ }^{5}$. "

Homme homérique, entre l'air et la pierre. Constamment "immobile à grands pas". Ne voyant aucun affect " lisible " que son visage eût exprimé, je comprenais que la " puissance d'être affecté " n'avait, chez lui, aucun signe mimique à produire. Elle traversait, elle portait et emportait tout son corps, tous ses gestes, son être entier et, même, l'espace qu'il créait autour de lui. Son affection - à tous les sens du mot - pour le taureau 
et le toreo, on la voyait dans l'ensemble de sa danse, jusque dans cette folie apparente qui le faisait attendre sans bouger, les deux jambes droites, face à la charge mortelle, et sans dévier, jusqu'à se faire, comme on dit si bien, prendre. José Tomás demeure impersonnel dans l'arène, par modestie et par grandeur. Il regarde souvent vers le sable. Ce n'est pas lui qui est ému, c'est sa danse qui consiste, dans la rencontre avec l'animal, à émouvoir ou " commouvoir l'air " tout entier dans l'arène immense : " en el aire conmovido ", ainsi que l'écrivait Federico García Lorca dans le premier poème de son Romancero gitano ${ }^{6}$. Sa concentration même tient à ce que Deleuze disait de l'émotion : qu'elle ne dit pas je, qu'elle est de l'ordre de l'événement et non de quelque subjectivité "personnelle ${ }^{7}$ ». S'il y a chorégraphie, elle est dictée non par un principe d'auteur ou, même, d' « artiste " - classique ou baroque, puriste ou " trémendiste " - mais, simplement, par l'éthique d'un digne placement de l'homme en face de ses deux morts à faire danser ensemble.

Il y a aussi les heures d'agonie. La danse coupée nette en une seule volte-face. Le 24 avril 2010, dans les arènes d'Aguascalientes, au Mexique, le taureau Navegante infligea au torero, en un retournement de tête d'à peine un quart de seconde, une cornada extrêmement grave. "Le sang giclait à gros bouillons de sa cuisse gauche ", écrivit Agustín Morales Padilla. " La corne a tranché la veine saphène et la fémorale. " Le rapport médical du docteur Alfredo Ruiz Romero indiquait : "Il s'agit d'un coup de corne de trente centimètres dans le triangle de Scarpa. Il affecte l'artère et la veine fémorales, avec lésions vasculaires. » On demandait aux spectateurs, par haut-parleurs, de donner leur sang, puisque José Tomás se vidait entièrement du sien. " Les volontaires se pressaient pour donner... " Les journaux racontèrent, mois après mois, la très lente et douloureuse "récupération " du torero, à travers des opérations chirurgicales successives au Mexique puis en Espagne ${ }^{8}$.

Deux ans plus tard, le 10 mai 2012, José Tomás - dont les apparitions publiques, combats de taureaux mis à part, sont extrêmement rares - prononça un bref discours... d'hommage à Navegante, dont l'apparition continuait de hanter ses rêves : " Pourquoi t'es-tu retourné de façon si imprévisible ${ }^{9}$ ? ", se demandait-il. Et il osa imaginer tout haut la réponse du toro bravo. Mario Vargas Llosa composa, pour l'occasion, un hommage à cet hommage, dans lequel il écrivait notamment : "L'art du toreo surgit quand l'homme qui va combattre, armé seulement d'un chiffon rouge, doit, s'il ne veut pas périr haché par les cornes, dominer la science du leurre, danser, bouger, esquiver la charge et, en même temps, interpréter ce ballet de la survie, dessiner des figures, des images qui obligent le taureau à danser aussi, pour répondre à ses postures et ses feintes, tandis qu'il attaque le torero et tente de le tuer. [...] Dans le beau discours que vous, José Tomás, avez improvisé quand on vous a remis le VIe prix Paquiro, vous parliez à Navegante, le toro bravo qui avait été sur le point de vous tuer dans les arènes d'Aguascalientes. Vous lui disiez : "En piste, chacun se comporte comme il est, en piste on ne peut pas feindre, en piste tout est pour de vrai." Vous aviez raison, maestro de maestros. Dans les feintes et les leurres dont est fait le toreo, dans ces jeux d'ombres chinoises, de passes et de postures, une vérité crue, essentielle, surgit avec une force imparable ${ }^{10}$."

Pour qui veut faire danser la pensée, il faudra donc tenir compte de cette extrémité que constitue le fait de danser la mort en face. Il faudra aussi, pour cela, bouleverser son propre langage. On comprend aisément que le toreo, en tant que danse des moments et de mouvements décisifs - la corne ou la vie, la blessure ou l'esquive bien tournée -, ait pu servir de paradigme à une telle exigence poétique. Dans Langage tangage, Michel Leiris définissait ainsi (si l'on peut dire) le mot corrida : "Drame torride à douces cadences cruelles et à cris d'or ${ }^{11}$. " Quant au mot toro, il surgissait dans la page sous l'espèce de ce qu'en espagnol on appelle el otro, l'autre : "L'autre, rétif, traître et tueur, si trop tôt rodé $^{12}$. " En 1945, la prise de position poétique intitulée « De la littérature considérée 
comme une tauromachie " - et publiée en préface à la réédition de L'Âge d'homme énonçait l'exigence avec force et simplicité : « Donc, je rêvais corne de taureau. Je me résignais mal à n'être qu'un littérateur. Le matador qui tire du danger couru l'occasion d'être plus brillant que jamais et montre toute la qualité de son style à l'instant qu'il est le plus menacé : voilà ce qui m'émerveillait, voilà ce que je voulais être ${ }^{13}$."

Style - mot qui évoque immédiatement une pointe acérée - rime donc avec risque. Ce que montre aussi la danse du torero face à la bête, c'est que risque rime fondamentalement avec rythme : ce rythme où, tout à la fois, l'on s'exprime du plus profond et l'on s'expose au plus dangereux du monde extérieur, " dans la folie du jour ". C'est une condition tout à la fois formelle et existentielle que Michel Leiris n'aura jamais cessé d'interroger, depuis Miroir de la tauromachie ${ }^{14}$ en 1938 jusqu'à ses textes ultérieurs, inspirés par ses amis peintres ou sculpteurs. Par exemple, lorsqu'il formulait, à propos de Giacometti, le paradoxe des formes décisives en tant qu'elles sont des " masses" ou "blocs... d'intensités fuyantes " : "Scruter la masse immense d'inconnu que propose le dehors, donner corps à quelques parcelles de la féerie que l'on porte au-dedans, ce double désir - l'on ne peut en douter - est celui de tout peintre ou sculpteur digne de ce nom. Peinture et sculpture, en effet, posent ce problème préjudiciel, quand on ne les ampute pas de la figuration : comment agencer une image ou un objet qui soit, tout à la fois, une réalité convaincante par sa structure même, la transcription fidèle du motif souvenu ou inventé et l'expression d'un moment essentiellement fuyant de la subjectivité15 ?"

Comme il arrive qu'un torero dédie son combat ou qu'un cantaor dédie sa copla, l'écrivain Leiris voulut, en 1939, dédier L'Âge d'homme à son ami Georges Bataille (" qui est à l'origine de ce livre $\left.{ }^{16} »\right)$. Or celui-ci venait juste de publier, pour la revue Acéphale, un texte incandescent, "tauromachique " à sa façon : il s'intitulait " La pratique de la joie devant la mort ". On y trouve, dans une formule admirable, explicitement nietzschéenne, l'éloge de " celui qui danse avec le temps qui le tue ${ }^{17}$ ". Retour à Nietzsche, donc. Qui lui-même, à la fin des années 1880, avait voulu se rendre dans le Sud de la France - selon le témoignage de Resa von Schirnhofer ${ }^{18}$, qui l'accompagnait - pour voir de ses yeux ce qu'était un véritable combat de taureaux. L'année même, 1945, où Leiris écrivait sa "Littérature considérée comme une tauromachie ", Bataille publiait son livre - chaotique, exigeant, intense -, rédigé de février à août 1944, Sur Nietzsche : volonté de chance.

Que la chance finisse par prendre la place de la puissance nietzschéenne - ou, mieux, qu'elle soit considérée par Bataille comme la puissance même en tant que force ouverte à l'aléa -, voici qui nous renseigne enfin sur cette danse que le penseur disait éprouver au plus profond de lui : " [...] cette danse du cour en moi qui rit du fond du désespoir ${ }^{19}$ ", et que rien, cependant, " ne pourrait briser ". Cela revenait à intensifier encore la puissance en tant que " puissance d'être affecté ". Cela revenait aussi à insister toujours plus sur cette danse que suppose le jeu perpétuel du dedans et du dehors, et que Bataille nomme alors une " communication " : "Ce qui est chance pour l'individu est "communication", perte de l'un dans l'autre. La "communication" est "durée de la perte". Trouverai-je à la fin l'accent gai, assez fou - et la subtilité de l'analyse - pour raconter la danse autour du temps (Zarathoustra, La Recherche du temps perdu) ${ }^{20}$ ? " Au même moment, Bataille publia - pour L'Espagne libre - un texte admirable sur son expérience espagnole : texte qui semblait faire tout danser ensemble, dans une spirale où Goya tournoyait avec la mort du torero Granero, avec la vuelta des danseuses de flamenco et avec le « cri excédant, déchiré, prolongé ${ }^{21} »$ du vieux cantaor Diego Bermúdez.

Faire danser la pensée serait donc, dans cette perspective, ouvrir toutes ses propres raisons à l'émotion, voire à la commotion. Ouvrir sa propre condition existentielle à la " chance ", heureuse ou mortelle. Ainsi, danser sollicite le cœur. On s'essouffle, on s'exalte, 
on s'épuise à trop danser. Même le spectateur, assis dans les gradins, découvre cette pulsation pénible de cœur soulevé. Il est étonnant que cette dernière expression puisse appeler deux significations aussi antithétiques. Dire que l'on ressent un haut-le-cœur, c'est témoigner d'une répulsion, d'un dégoût, d'une nausée. Beaucoup ressentent cela, aujourd'hui plus que jamais, à l'égard de la tauromachie. Leiris, en 1934 - dans Grande fuite de neige -, s'est souvenu de sa première corrida. C'était en août 1926 et ce n'était, dit-il, qu'une " horrible tuerie ». Mais, ajoute-t-il, " loin d'en être écouré, je me sentis impatient d'en voir une où la bête serait, non plus bafouée, mais située à son juste niveau de sacrifice ou de tragédie ${ }^{22} "$. Histoire de transformer le haut-le-cœur en dignité, en ferveur devant les courages (mot qui vient de cœur, bien sûr) des protagonistes du combat, et pour lesquels on emploie une expression comme haut les cœurs.

Bataille, de son côté, profita de son emploi à la Bibliothèque nationale pour emprunter, entre le 8 mars et le 15 septembre 1933, un volume du Jahrbuch für Philosophie und phänomenologische Forschung, dirigé par Edmund Husserl, et dans lequel se trouvait l'étude magistrale, psychanalytique et existentielle d'Aurel Kolnai sur le « dégoût » (Ekel). Kolnai osait entrer dans l'impur et la matérialité de l'abjection à partir de ce que Heidegger, à propos de l'angoisse, avait en quelque sorte laissé dans l'abstrait. Il mettait surtout en avant l'ambivalence fondamentale du dégoût : c'est la phobie de toucher quelque chose qui, cependant, nous attire quoi qu'on en ait. "Le dégoût se détourne non seulement de son objet, mais encore d'une attirance du sujet pour celui-ci. [...] Sa logique interne comporte la possibilité d'une appréhension positive de l'objet - qu'il s'agisse de le toucher, de l'étreindre, de le consommer ${ }^{23}$ ", écrivait le philosophe hongrois. Faire danser la pensée ? Savoir traverser ce qui s'impose d'abord sous l'angle du dégoût, passer par-delà bien et mal. Puis savoir aller et venir entre les deux pôles, les deux façons d'être affecté. Faire valser les contradictions apparentes. Accepter jusqu'au bout - vouloir - cette fragilité où nous place notre puissance d'être affectés. 


\section{NOTES}

1. Friedrich Nietzsche, Le Gai Savoir (1882-1887), dans OEuvres philosophiques complètes, t. V, G. Colli et M. Montinari (éd.), M. de Gandillac (trad. fr.), Paris, Gallimard, 1982, p. 293.

2. Nietzsche, 1982, cité n. 1, p. 49.

3. Nietzsche, 1982, cité n. 1, p. 59.

4. Gilles Deleuze, Nietzsche et la philosophie (1962), Paris, Presses universitaires de France, 1997, p. 70.

5. Jacques Durand, José Tomás - Madrid, Barcelone, 2008, Arles, Actes Sud, 2008, p. 51 et 55.

6. Federico García Lorca, Romancero gitano (1928), Obras completas, I (1954), A. del Hoyo (éd.), Madrid, Aguilar, 1987, p. 393.

7. Gilles Deleuze, «La peinture enflamme l'écriture » (1981), dans Deux régimes de fous. Textes et entretiens, 1975-1995, D. Lapoujade (éd.), Paris, Les Éditions de Minuit, 2003, p. 172.

8. Agustín Morales Padilla, "José Tomás » (2012), dans Dialogue avec Navegante, A. Martin (trad. fr.), Vauvert, Au Diable Vauvert, 2013, p. 91, 99 et 102.

9. José Tomás, "Navegante » (2012), F. Zumbiehl (trad. fr.), dans Morales Padilla, 2013, cité n. 8, p. 15-21.

10. Mario Vargas Llosa, "Monologue du taureau (face à José Tomás) » (2012), dans Morales Padilla, 2013, cité n. 8, p. 29 et 32 .

11. Michel Leiris, Langage tangage, ou Ce que les mots me disent (1985), Paris, Gallimard, 1995, p. 19.

12. Leiris, 1995, cité n. 11, p. 59.

13. Michel Leiris, L'Âge d'homme (1939), précédé de De la littérature considérée comme une tauromachie (1945-1946), Paris, Gallimard, 1973, p. 12.

14. Michel Leiris, Miroir de la tauromachie (Paris, 1938), Montpellier, Fata Morgana, 2013.

15. Michel Leiris, "Alberto Giacometti, en timbre-poste ou en médaillon » (Paris, 1962), republié dans Brisées, Paris, Gallimard, 1992, p. 275-276.

16. Leiris, 1973, cité n. 13, p. 7.

17. Georges Bataille, «La pratique de la joie devant la mort » (1939), dans CEuvres complètes, t. I, Paris, Gallimard, 1970, p. 554

18. Curt Paul Janz, Nietzsche, Biographie, t. III. Les dernières années du libre philosophe, la maladie (1978), Pierre Rusch et Michel Vallois (trad. fr.), Paris, Gallimard, 1985, p. 35 [éd. orig. : Friedrich Nietzsche. Biographie, 3. Die Jahre des Siechtums. Dokumente, Quellen und Register, Munich, Carl Hanser-Verlag, 1978-1979].

19. Georges Bataille, Sur Nietzsche : volonté de chance (1945), CEuvres complètes, t. VI, Paris, Gallimard, 1973, p. 96.

20. Bataille, 1973, cité n. 19, p. 150.

21. Georges Bataille, "À propos de Pour qui sonne le glas d'Ernest Hemingway » (1945), reproduit dans M. Surya (dir.), Georges Bataille, une liberté souveraine, cat. exp. (Orléans, Médiathèque, 1997-1998), Orléans / Fourbis-Ville d'Orléans, 1997, p. 41-47 (le texte reproduit dans OEuvres complètes, t. XI, Paris, Gallimard, 1988, p. 25-26, étant incomplet). Voir Georges Didi-Huberman, "L'œil de l'expérience ", dans Carlo Ginzburg, MarieJosé Mondzain, Michel Deguy et al., Vivre le sens. Centre Roland-Barthes, Paris, Éditions du Seuil, 2008 p. 147-177.

22. Michel Leiris, Grande fuite de neige (Paris, 1934), Montpellier, Fata Morgana, 1992, p. 9-10.

23. Aurel Kolnai, Le Dégoût, Paris, Agalma, 1997, p. 42-43 [éd. orig. : « Der Ekel », dans Edmund Husserl (dir.), Jahrbuch für Philosophie und phänomenologische Forschung, 1929]. 